\title{
Correlation of Intracranial Volume With Head Surface Volume in Patients With Multisutural Craniosynostosis
}

\author{
Karan R.R. Ramdat Misier, BSc, ${ }^{*}$ Richard W.F. Breakey, MRCS, ${ }^{\dagger}$ \\ Cornelia J.J.M. Caron, MD, DMD, ${ }^{*}$ Silvia Schievano, MEng, PhD, ${ }^{\dagger}$ \\ David J. Dunaway, FDSRCS, FRCS (Plast), ${ }^{\dagger}$ Maarten J. Koudstaal, MD, PhD, ${ }^{* \dagger \ddagger}$ \\ Owase N.U. Jeelani, MPhil, FRCS (NeuroSurg), ${ }^{\dagger}$ and Alessandro Borghi, MEng, PhD ${ }^{\dagger}$
}

\begin{abstract}
Intracranial volume (ICV) is an important parameter for monitoring patients with multisutural craniosynostosis. Intracranial volume measurements are routinely derived from computed tomography (CT) head scans, which involves ionizing radiation. Estimation of ICV from head surface volumes could prove useful as 3D surface scanners could be used to indirectly acquire ICV information, using a non-invasive, non-ionizing method.
\end{abstract}

Pre- and postoperative 3D CT scans from spring-assisted posterior vault expansion (sPVE) patients operated between 2008 and 2018 in a single center were collected. Patients were treated for multisutural craniosynostosis, both syndromic and non-syndromic. For each patient, ICV was calculated from the CT scans as carried out in clinical practice. Additionally, the $3 \mathrm{D}$ soft tissue surface volume (STV) was extracted by $3 \mathrm{D}$ reconstruction of the CT image soft tissue of each case, further elaborated by computer-aided design (CAD) software. Correlations were analyzed before surgery, after surgery, combined for all patients and in syndrome subgroups.

Soft tissue surface volume was highly correlated to ICV for all analyses: $r=0.946$ preoperatively, $r=0.959$ postoperatively, and $r=0.960$ all cases combined. Subgroup analyses for Apert, Crouzon-Pfeiffer and complex craniosynostosis were highly significant as well $(P<0.001)$.

In conclusion, 3D surface model volumes correlated strongly to $\mathrm{ICV}$, measured from the same scan, and linear equations for this correlation are provided. Estimation of ICV with just a 3D surface model could thus be realized using a simple method, which does not

From the *Department of Oral and Maxillofacial Surgery, Erasmus University Medical Center, Rotterdam, the Netherlands; †Craniofacial Unit, Great Ormond Street Hospital for Children, London, United Kingdom; and ‡Department of Craniofacial Malformations, Karolinska University Hospital, Stockholm, Sweden.

Received September 28, 2019.

Accepted for publication January 14, 2020.

Address correspondence and reprint requests to Karan R.R. Ramdat Misier, BSc, Department of Oral and Maxillofacial surgery, Erasmus MC Rotterdam, Doctor Molewaterplein 40, 3015 GD Rotterdam, the Netherlands; E-mail: k.ramdatmisier@erasmusmc.nl

The authors report no conflicts of interest.

Supplemental digital contents are available for this article. Direct URL citations appear in the printed text and are provided in the HTML and PDF versions of this article on the journal's Web site (www.jcraniofacialsurgery.com).

Copyright (C) 2020 by Mutaz B. Habal, MD

ISSN: $1049-2275$

DOI: $10.1097 /$ SCS.0000000000006372 require radiations and therefore would allow closer monitoring in patients through multiple acquisitions over time.

Key Words: 3D imaging, craniosynostosis, intracranial volume

(J Craniofac Surg 2020;31: 1445-1448)

raniosynostosis is best described as the premature fusion of one or more calvarial sutures. ${ }^{1}$ This can cause multiple problems, including raised intracranial pressure, visual impairment and possible neuro-developmental delay. Treatment involves skull surgery, aiming to enlarge the volume of the cranial vault. ${ }^{2}$ Posterior vault expansion is a common surgical procedure which entails the expansion of the posterior skull; metal springs can be used to promote calvarial augmentation in a procedure called springassisted posterior vault expansion (sPVE) (Fig. 1). ${ }^{3}$

Measurement of intracranial volume (ICV) provides important information in the monitoring of craniosynostosis patients as, albeit not a direct quantification of intracranial pressure, it may provide information on the space available inside the skull and the postsurgical volume increase. ${ }^{4-6}$ In order to calculate a patient's ICV, an imaging technique yielding a 3-dimensional (3D) view of the intracranial area, such as computed tomography (CT) or magnetic resonance imaging (MRI), is required. ${ }^{4}$ Yet, MRI is not routinely used in the evaluation of craniosynostosis because of its timeconsuming nature and the need for sedation in children. ${ }^{7,8}$ The use of CT is limited due to its accompanying radiation dose, as opposed to alternative techniques such as 3D-photogrammetry which, however, do not entail imaging of the intracranial area. ${ }^{9}$ 3D-photogrammetry has increasingly been used in craniofacial imaging, since it has proven to produce valid 3D surface images, which are anthropometrically and volumetrically analogous to CT images and lack the associated radiation dose. ${ }^{10-12}$ A technique for reliably estimating ICV from 3D-photogrammetry surface models would allow
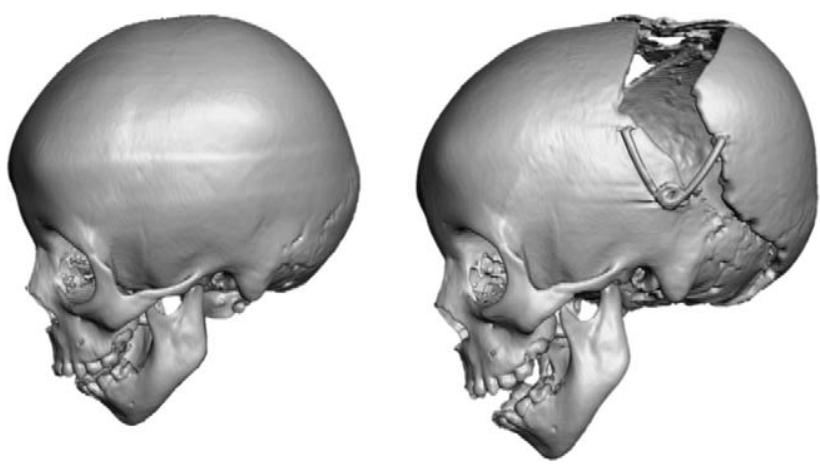

FIGURE 1. Preoperative (left) and postoperative (right) 3D models of a patient that underwent spring-assisted posterior vault expansion. 
estimation of change in ICV in a larger population and would be particularly welcome in the pediatric population, a population in which scanning may take several times and unnecessary radiation dose should be avoided. ${ }^{13}$

In this study, we aimed to measure ICV and the total soft tissue volume of 3D surface models (STV), before and after sPVE, to investigate a possible correlation between the 2 volumes in patients with multisutural craniosynostosis.

\section{METHODS}

\section{Patient Selection}

Pre- and postoperative scans of consecutive patients with syndromic and complex craniosynostosis, who underwent sPVE at Great Ormond Street Hospital for Children (London, UK) between 2008 and 2018, were retrospectively collected. Patients were classified under complex craniosynostosis when multisutural synostosis was present, without an accompanying syndrome diagnosis. Scans were included if the patient had a diagnosis of multisutural craniosynostosis, and both the preoperative and postoperative scans were available. Scans with a slice thickness greater than $3 \mathrm{~mm}$ were excluded.

\section{Data Processing and Volume Calculation}

Measurements for ICV were performed on 3D CT scans. An extensive description of the measurement methods can be found in Breakey et al. ${ }^{4,14}$ In short, FSL (FMRIB Analysis Group, Oxford, United Kingdom) was used for ICV automatic calculation ${ }^{15}$; when this method failed, a semi-automatic method was adopted using Simpleware ScanIP (Synopsys Inc, Mountain View, CA), a voxelbased method for creating a specific mask, where areas out of interest can be manually excluded ${ }^{14}$ (Fig. 2). Intracranial volume is obtained by calculation of the voxel information within the thresholded mask.

For STV, DICOM files of the same CT scan were imported into Simpleware ScanIP, where a soft tissue 3D surface was thresholded, using 'Mask flood fill' and 'Unpaint' functions to exclude unwanted regions. The stereolithography (STL) 3D models were imported in to Autodesk Meshmixer (Autodesk Research, Toronto, Canada), where they were cut with a plane through the nasion (deepest portion of the nasofrontal groove in the face midline) and both tragions (notch above the tragus) ${ }^{16}$ (Fig. 3). After the planar cut, the remainders of the ears were cut out of the model and the voids closed using the 'Bridge' and 'Smooth MVC' functions (Fig. 4). The volume of the resulting 3D model was retrieved as STV.

\section{Statistical Analysis}

Correlations between STV and ICV were studied by Pearson's correlation coefficient $(r)$, calculated using $R$ ( $R$ foundation for Statistical Computing, Vienna, Austria). Linear equations for the found correlations were calculated and visualized using Microsoft Excel (Version 2010; Microsoft Redmond, WA).
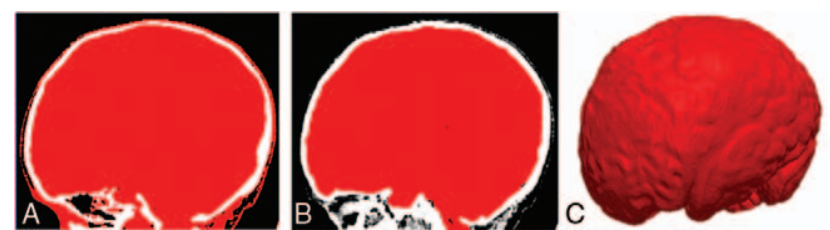

FIGURE 2. Measurement of the intracranial volume. A: thresholding of the model. B: exclusion of thresholded areas outside the region of interest. C: 3D visualisation of the intracranial area.
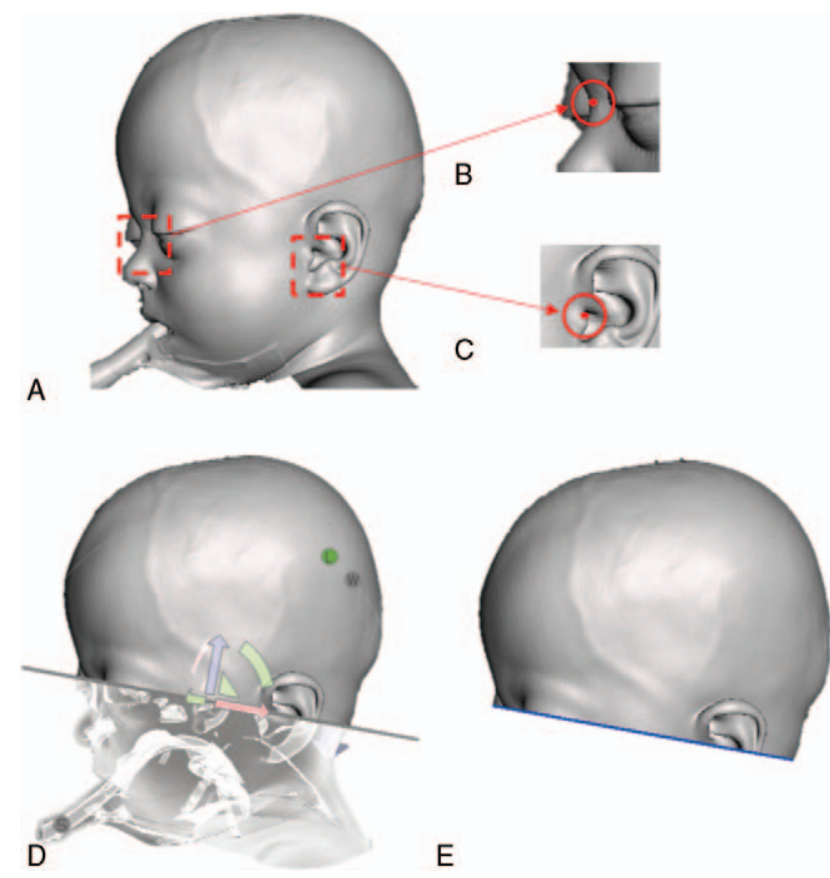

FIGURE 3. Planar cut in the soft tissue model. A: Soft-tissue model. B: Nasion; C: Tragion; D: Cutting plane; E: region of interest resulting from the planar cut.

\section{RESULTS}

Sixty-eight pre- and postoperative CT scans of 34 syndromic and complex craniosynostosis patients were collected. Mean age at time of the preoperative CT scan was $2.1 \pm 1.8$ years and at time of the postoperative scan $3.3 \pm 2.3$ years. 19 of the 34 patients were male $(56 \%)$. Patient diagnoses included Apert $(n=8)$, Crouzon-Pfeiffer $(\mathrm{n}=12)$, Complex craniosynostosis $(\mathrm{n}=11)$, Noonan $(\mathrm{n}=1)$, ERF $(\mathrm{n}=1)$ and Muenke $(\mathrm{n}=1)$. (Supplemental Digital Content, Table 1, http://links.lww.com/SCS/B283).

On average, STV was $33.2 \%$ larger than ICV. In Supplemental Digital Content, Table 2, http://links.lww.com/SCS/B284 preoperative, postoperative and combined Pearson's correlation coefficients are displayed for the largest patient groups, being Crouzon-Pfeiffer, Apert and Complex craniosynostosis, and for all patients combined. Preoperatively, the combined correlation coefficient was $r=0.946$; postoperatively the combined correlation coefficient was $r=0.959$. For all patients combined, the correlation coefficient was $r=0.961$ (Fig. 5).

In Supplemental Digital Content, Table 3, http://links.lww.com/ SCS/B285 linear equations for retrieving ICV from STV are shown. When STV is given as $\mathrm{x}$ in $\mathrm{cm}^{3}$, the equation provides $\mathrm{ICV}$ in $\mathrm{cm}^{3}$.

\section{DISCUSSION}

Significant correlations were found between the volume of soft tissue 3D surface models and the intracranial volume, both in syndromic and complex craniosynostosis patients grouped together

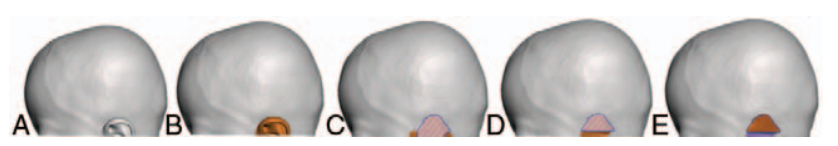

FIGURE 4. Removal of the ear lobe. A: region of interest of the head model. B: Selection of the ear lobe. C: Removal of the ear lobe and selection of resulting borders. D: 'Bridging' of the open surface. E: Filling of the void after bridging. 

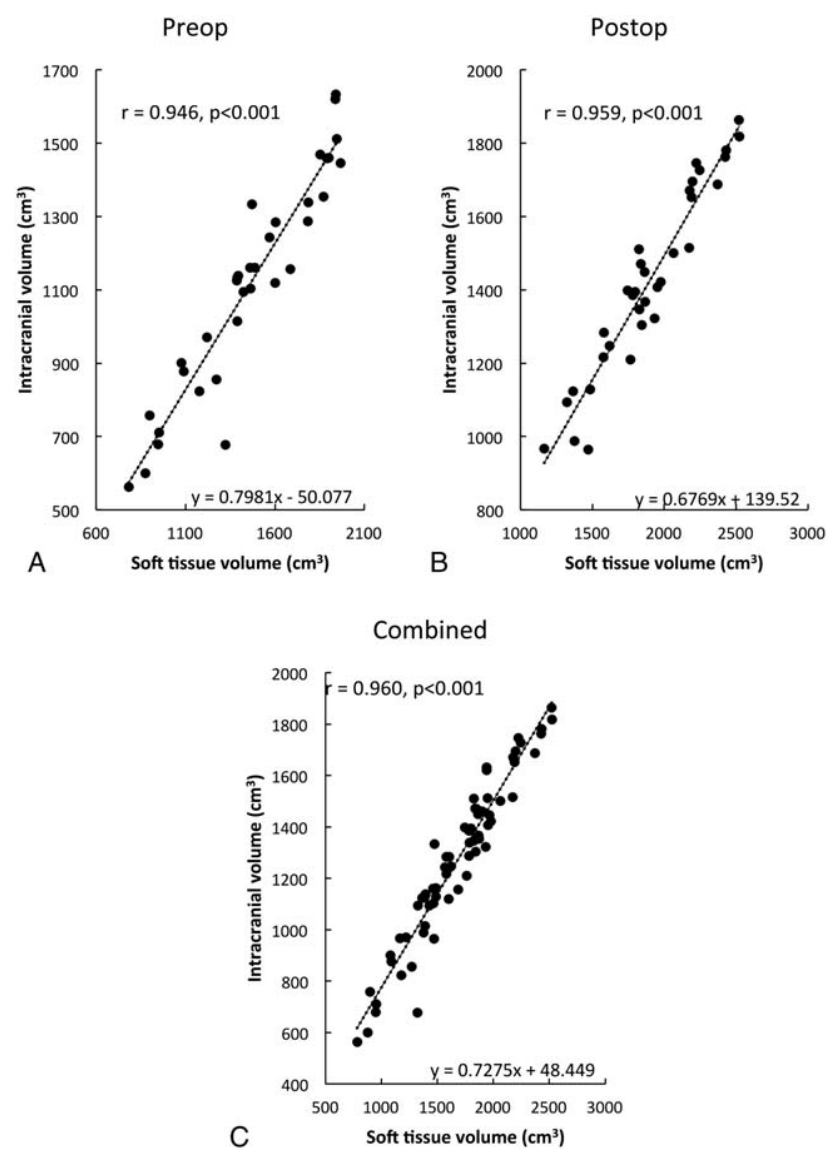

FIGURE 5. Correlation plots for the preoperative (A), postoperative (B) and combined $(C)$ correlations between the soft tissue volume and intracranial volume. Dotted line represents the linear equation, which is shown in the bottom right corner.

and in separate groups for Crouzon-Pfeiffer, Apert and complex craniosynostosis. Separate preoperative and postoperative correlations were highly significant as well. This indicates that STV is a good indicator for ICV, both before and after skull surgery (sPVE in the current study). All volumes were extracted from the same CT scans and processing of the soft tissue 3D-meshes occurred in a consistent and reproducible way, using a cutting plane previously described in the literature. ${ }^{17,18}$ The software used for the STV calculation - Autodesk Meshmixer - is freely available for download. Using the reported equations, future studies using 3D-photogrammetry could calculate an estimation of ICV by processing the $3 \mathrm{D}$ model as described and entering the STV into the equation.

McKay et al have described the validity of 3D-photogrammetry against CT. The authors compared the cranial vault volume of CT scans to the volume of 3D images derived from 3D-photogrammetry. Measured volumes were highly correlated in the two imaging techniques, indicating that volumes derived from CT scans, as used in the current study, are interchangeable to volumes derived from 3D-photogrammetry, for which we presume our study is useful. ${ }^{10}$

Van Veelen et al found a good correlation for the total volume of $3 \mathrm{D}$-models and ICV in 10 sagittal synostosis patients; ${ }^{19}$ the authors used 3D-photogrammetry scans which were acquired on the same day as CT scans, correlated those volumes to ICV and found a Pearson's correlation coefficient of $>0.86$. The cutting plane, running through one tragus and 2 outer cantii, was similar to the plane used in this study. ${ }^{19}$ In the present study, we expand these outcomes in a larger group of patients for multisutural craniosynostosis, both syndromic and nonsyndromic and both preoperatively and postoperatively.

The STV used in the present study has been used to analyze the outcome of sagittal synostosis correction in the past: Tenhagen et al quantified outcomes of spring-assisted surgical correction of sagittal synostosis. ${ }^{18}$ Rodriguez-Florez also used the same cutting plane for calculating the head volume under a soft tissue $3 \mathrm{D}$ model. ${ }^{17}$ The authors calculated the volumes using the vascular modelling toolkit (VMTK, Orobix, Bergamo, Italy) in combination with MATLAB (The MathWorks Inc, Natick, MA), thus requiring extra software. The volumes in our study were easily retrieved in Autodesk Meshmixer, as they can automatically be displayed after processing, and do not require knowledge or purchase of extra software.

The main limitation to this study is the lack of validation of the soft tissue volumes in images derived from a 3D-scanner; the used models and corresponding volumes were all derived from CT images. However, multiple studies have addressed the conformity and interchangeability of 3D images derived from CT scans and 3Dscanning. ${ }^{10,11,19,20}$ Therefore, we assume that the CT soft tissue models used in this study are a valid representation of 3D models derived from 3D-photogrammetry. Furthermore, the mentioned limit may be considered a strength, as the volumes are derived from exactly the same scan, preventing a possible discrepancy between scans derived from different imaging techniques.

For further confirmation of the measurements, a future study could prospectively include 3D-scans of a large group of patients who underwent a CT scan during the same imaging session. Hereafter, the described processing method can be applied to images of 3D-photogrammetry for further validation of the obtained correlations.

\section{CONCLUSION}

In this study, we have displayed highly significant correlations between the total volume of 3D surface models and the intracranial volume, measured from the same CT scan in multisutural craniosynostosis patients. We provide linear equations which can be used to calculate an estimation of the intracranial volume using the soft tissue volume. The current results may be of interest when $3 \mathrm{D}$ photogrammetry is used in craniofacial imaging and an estimation of intracranial volume from these images is desired.

\section{REFERENCES}

1. Johnson D, Wilkie AOM. Craniosynostosis. Eur J Hum Genet 2011;19:369-376

2. Panchal J, Uttchin V. Management of craniosynostosis. Plast Reconst Surg 2003;111:2032-2048quiz 49

3. De Jong T, Van Veelen MLC, Mathijssen IMJ. Spring-assisted posterior vault expansion in multisuture craniosynostosis. Child's Nerv Syst 2013;29:815-820

4. Breakey RWF, Knoops PGM, Borghi A, et al. Intracranial volume and head circumference in children with unoperated syndromic craniosynostosis. Plast Reconstr Surg 2018;142:708e-717e

5. Derderian CA, Wink JD, McGrath JL, et al. Volumetric changes in cranial vault expansion: comparison of fronto-orbital advancement and posterior cranial vault distraction osteogenesis. Plast Reconstr Surg 2015;135:1665-1672

6. Serlo WS, Ylikontiola LP, Lähdesluoma N, et al. Posterior cranial vault distraction osteogenesis in craniosynostosis: estimated increases in intracranial volume. Child's Nerv Syst 2011;27:627-633

7. Kim HJ, Roh HG, Lee IW. Craniosynostosis: updates in radiologic diagnosis. J Korean Neurosurg Soc 2016;59:219-226

8. Lawson GR. Controversy: sedation of children for magnetic resonance imaging. Arch Dis Child 2000;82:150-153

9. Almohiy H. Paediatric computed tomography radiation dose: a review of the global dilemma. World J Radiol 2014;6:1-6 
10. McKay DR, Davidge KM, Williams SK, et al. Measuring cranial vault volume with three-dimensional photography: a method of measurement comparable to the gold standard. J Craniofac Surg 2010;21:1419-1422

11. Mendonca DA, Naidoo SD, Skolnick G, et al. Comparative study of cranial anthropometric measurement by traditional calipers to computed tomography and three-dimensional photogrammetry. J Craniofac Surg 2013;24:1106-1110

12. Wong JY, Oh AK, Ohta E, et al. Validity and reliability of craniofacial anthropometric measurement of 3D digital photogrammetric images. Cleft Palate Craniofac J 2008;45:232-239

13. Seeberger R, Hoffmann J, Freudlsperger C, et al. Intracranial volume (ICV) in isolated sagittal craniosynostosis measured by $3 \mathrm{D}$ photocephalometry: a new perspective on a controversial issue. J Craniomaxillofac Surg 2016;44:626-631

14. Breakey W, Knoops PGM, Borghi A, et al. Intracranial volume measurement: a systematic review and comparison of different techniques. J Craniofac Surg 2017;28:1746-1751

15. Jenkinson M, Beckmann CF, Behrens TE, et al. Fsl. Neuroimage 2012;62:782-790
16. Guyuron B. Precision rhinoplasty: part I. The role of life-size photographs and soft-tissue cephalometric analysis. Plast Reconstr Surg 1988;81:489-499

17. Rodriguez-Florez N, Bruse JL, Borghi A, et al. Statistical shape modelling to aid surgical planning: associations between surgical parameters and head shapes following spring-assisted cranioplasty. Int $J$ Comput Assist Radiol Surg 2017;12:1739-1749

18. Tenhagen M, Bruse JL, Rodriguez-Florez N, et al. Three-dimensional handheld scanning to quantify head-shape changes in spring-assisted surgery for sagittal craniosynostosis. J Craniofac Surg 2016;27: $2117-2123$

19. van Veelen M-LC, Jippes M, Carolina J-CA, et al. Volume measurements on three-dimensional photogrammetry after extended strip versus total cranial remodeling for sagittal synostosis: a comparative cohort study. J Cranio-Maxillofac Surg 2016;44: $1713-1718$

20. Fourie Z, Damstra J, Gerrits PO, et al. Evaluation of anthropometric accuracy and reliability using different three-dimensional scanning systems. Forensic Sci Int 2011;207:127-134

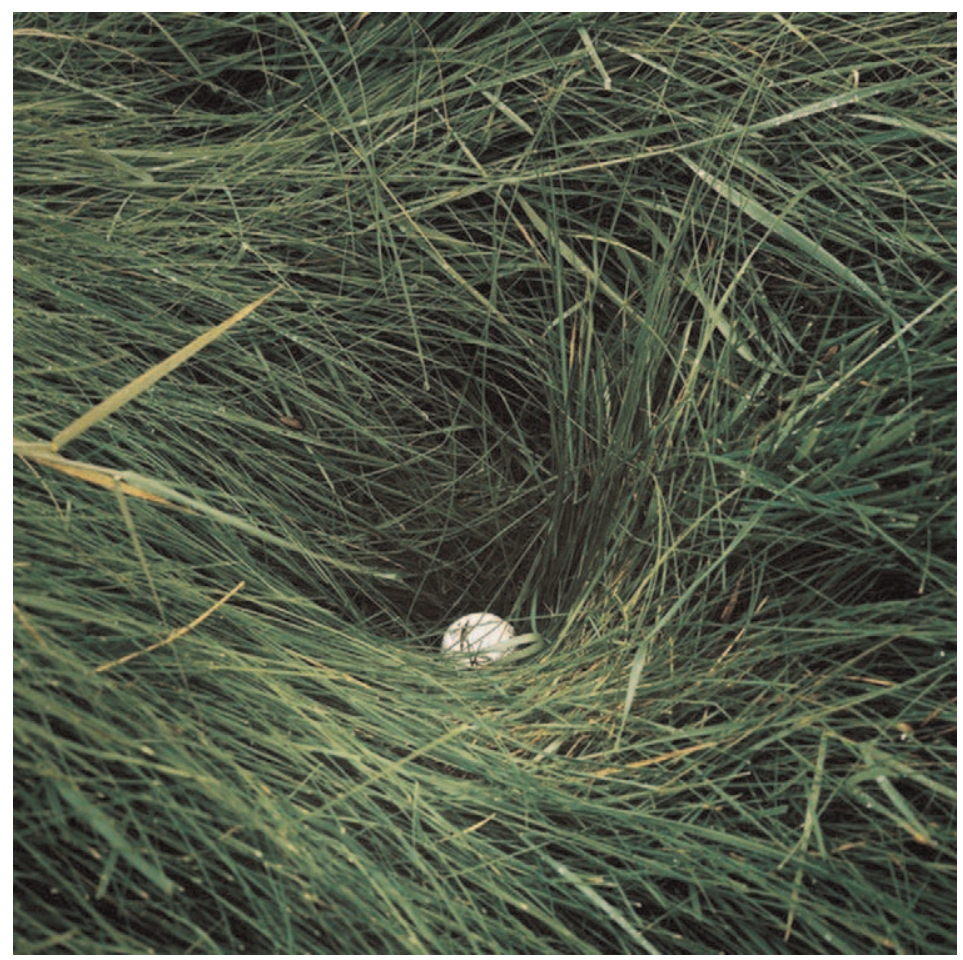

